\title{
RESULTADOS DO TRATAMENTO CIRÚRGICO DA DOENÇA DO REFLUXO GASTROESOFÁGICO EM CRIANÇAS COM E SEM AFECÇÕES NEUROLÓGICAS CONCOMITANTES
}

\section{Results of surgical treatment of gastroesophageal reflux disease in children with and without concomitant neurological affections}

\author{
José Lúcio Martins MACHADO, Bonifácio Katsunori TAKEGAWA, Érika Veruska de O. Paiva ORTOLAN, \\ Antonio Marcos RODRIGUES, Ana Maria FIORAVANTE
}

ABCDDV/524

\begin{abstract}
Machado JLM, Takegawa BK, Ortolan EVOP, Rodrigues AM, Fioravante AM. Resultados do tratamento cirúrgico da doença do refluxo gastroesofágico em crianças com e sem afecções neurológicas concomitantes. ABCD Arq Bras Cir Dig 2007;20(1):45-50.

RESUMO - Racional - Crianças com distúrbios neurológicos têm maior incidência de refluxo e, em geral, não apresentam melhora da sintomatologia com tratamento clínico, necessitando de intervenção cirúrgica. Objetivo - Comparar os resultados da operação antirefluxo em crianças normais e com comprometimento neurológico, identificando as principais complicações e causas de reoperação. Métodos - Cento e vinte crianças portadores de refluxo foram distribuídas em dois grupos de estudo: Grupo I - 60 crianças normais; Grupo II - 60 crianças com comprometimento neurológico. Exame contrastado do esôfago, estômago e duodeno, endoscopia digestiva alta com biópsia, pHmetria esofágica de 24 horas e cintilografia foram os exames utilizados no diagnóstico e na avaliação da eficácia da operação antirefluxo. Todos os pacientes operados eram refratários ao tratamento clínico. O procedimento cirúrgico antirefluxo realizado foi predominantemente a fundoplicatura de Lind, sendo associada à gastrostomia em $55 \%$ dos pacientes do Grupo II. Resultados - No Grupo II a indicação cirúrgica foi significantemente mais precoce que no Grupo I. A principal causa de indicação cirúrgica entre neuropatas foi o alto comprometimento do desenvolvimento neuropsíquico-motor e as pneumonias de repetição. O tempo de internação, as reoperações e a necessidade de dilatações esofágicas no pós-operatório foi maior no Grupo II. Ocorreram três óbitos no pós-operatório tardio no Grupo II (sepse e infecção respiratória grave). Conclusão - O tratamento cirúrgico adotado foi satisfatório para o tratamento cirúrgico do refluxo nos dois grupos de pacientes. Porém, torna-se necessário o aprofundamento dos estudos acerca da população de crianças neuropatas portadoras de refluxo, uma vez que estas respondem de forma menos favorável ao procedimento cirúrgico, principalmente no que se refere às taxas de mortalidade, recorrência dos sintomas respiratórios, índice de reoperações e gravidade das complicações pós-operatórias.

DESCRITORES - Refluxo gastroesofágico. Esofagite péptica. Manifestações neurológicas.
\end{abstract}

\section{INTRODUÇÃO}

A doença do refluxo gastroesofágico (DRGE) é entidade clínica comum na população pediátrica, podendo causar importantes distúrbios nutricionais e respiratórios tais como retardo no crescimento, esofagite, aspirações, pneumonias recorrentes e apnéias ${ }^{13}$.

A associação entre DRGE e doenças do sistema nervoso central tem sido demonstrada em muitos estudos clínicos $^{1,18}$. Crianças com distúrbios neurológicos têm maior incidência de DRGE e, na maioria dos casos, não apresentam melhora da sintomatologia com tratamento clínico, necessitando de intervenção cirúrgica.

A causa da DRGE em neuropatas não está bem definida. Possíveis explicações incluem: posição supina predominante, aumento da pressão intra-abdominal devido à escoliose, espasticidade da musculatura abdominal,

Trabalho realizado na Disciplina de Cirurgia Pediátrica do Departamento de Cirurgia e Ortopedia da Faculdade de Medicina de Botucatu - UNESP, Botucatu, SP, Brasil.

Endereço para correspondência: José Lúcio Martins Machado, e-mail: jluciomm@yahoo.com.br aerofagia, constipação e dismotilidade gastroesofágica ${ }^{6,7}$.

$\mathrm{O}$ procedimento anti-refluxo mais freqüentemente realizado é a fundoplicatura pela técnica de Nissen, sendo que nos pacientes com comprometimento neurológico há elevada taxa de morbimortalidade. A casuística dos autores deste trabalho aponta para a utilização do procedimento mais difundido em nosso meio, que é a fundoplicatura parcial de Lind, especialmente para a população de pacientes com DRGE portadores de neuropatias.

O objetivo deste estudo é comparar os resultados da operação anti-refluxo em crianças acometidas por DRGE, com e sem comprometimento neurológico, e identificar as principais complicações e causas de reoperações.

\section{MÉTODOS}

Em estudo retrospectivo de 10 anos foram analisados 120 pacientes com DRGE, submetidos ao tratamento cirúrgico no Hospital das Clínicas da Faculdade de Medicina de Botucatu - Unesp, Botucatu, SP, Brasil. Esses pacientes foram distribuídos em dois grupos, sendo que 
o primeiro (Grupo I) foi constituído de 60 pacientes com DRGE, sem alteração neurológica e o segundo (Grupo II) de 60 pacientes com DRGE, com alteração neurológica comprovada clinicamente.

Os pacientes foram caracterizados de acordo com as seguintes variáveis: sexo, faixa etária, sinais e sintomas prevalentes, diagnósticos concomitantes, evolução ponderal, exames complementares, tempo de tratamento clínico prévio à operação, medicamentos utilizados, tipo de operação, tempo de internação, complicações no pósoperatório imediato e tardio e reoperações.

Os resultados foram analisados de acordo com teste do qui-quadrado $\left(\chi^{2}\right)$. Para as comparações foi considerado o nível de significância $P<0,05$.

\section{RESULTADOS}

\section{Quanto ao sexo e faixa etária}

Não houve diferença entre os grupos, sendo que no Grupo I, $35(58,4 \%)$ eram pacientes do sexo masculino e $25(41,6 \%)$ do sexo feminino e no Grupo II, $31(51,7 \%)$ do sexo masculino e $29(48,3 \%)$ do sexo feminino.

Em ambos os grupos a faixa etária predominante foi dos lactentes (51,6\% do Grupo I e 58,3\% do Grupo II), sendo que no Grupo I, 31,6\% dos pacientes tinham entre 1 e 2 anos de idade e no Grupo II, 26,7\% tinham entre 6 meses e 1 ano.

A análise dos resultados apontam para diferença estatisticamente significante na distribuição dos pacientes nas faixas etárias predominantes $(P<0,01)$. Os dados são apresentados na Figura 1.

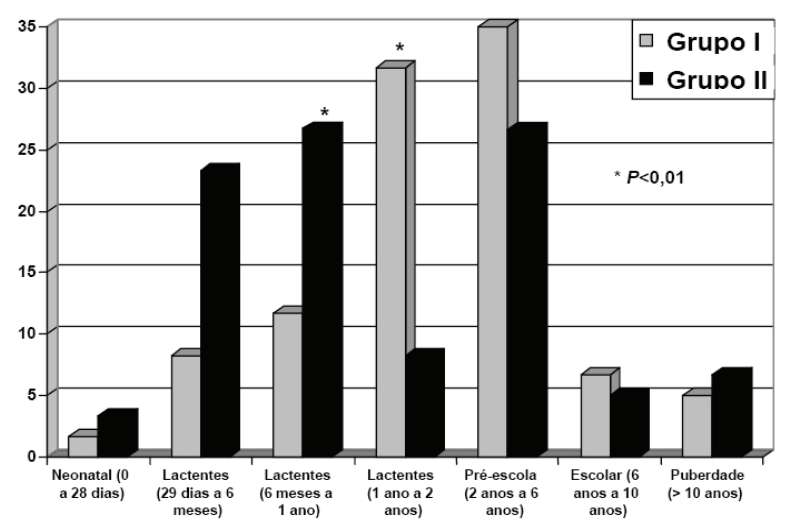

FIGURA 1 - Distribuição dos pacientes segundo a faixa etária

\section{Sinais e sintomas predominantes}

Os sinais e sintomas mais freqüentes no Grupo I foram: vômitos $(75 \%)$, regurgitação $(61,7 \%)$, tosse noturna $(61,7 \%)$ e pneumonia $(56,7 \%)$. No Grupo II foram: pneumonia $(90 \%)$, vômitos $(60 \%)$, regurgitação $(53,3 \%)$ e convulsão (46,7\%). Os dados são apresentados na Figura 2.

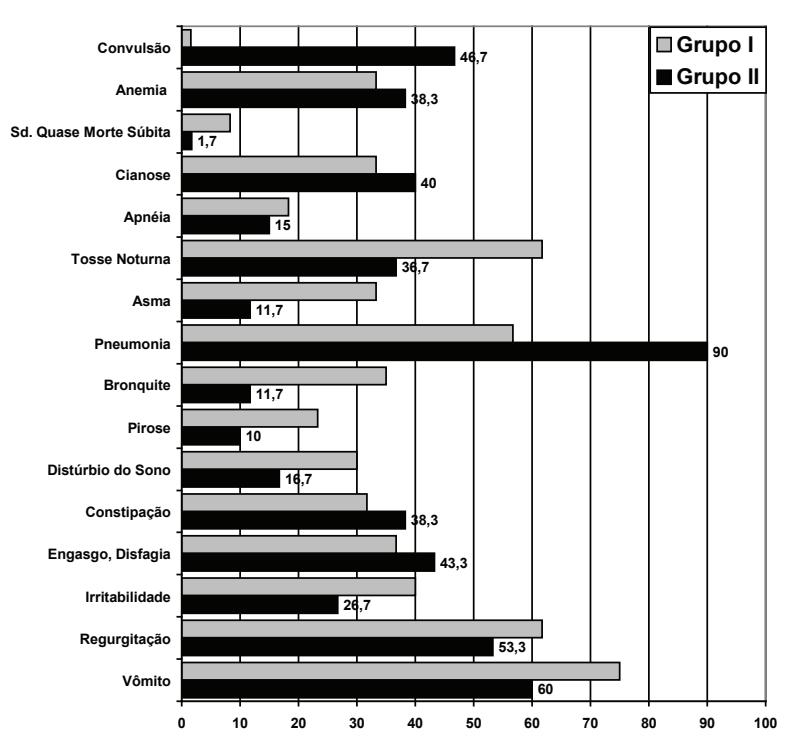

FIGURA 2 - Distribuição dos pacientes segundo a freqüência dos sinais e sintomas de refluxo gastroesofágico

\section{Diagnósticos concomitantes}

A hérnia de hiato esteve presente em 15 pacientes (25\%) no Grupo I e em 10 pacientes (16,9\%) no Grupo II. Outras afecções concomitantes foram: atresia de esôfago, fístula traqueoesofágica e as cardiopatias congênitas.

O diagnóstico das alterações neurológicas presentes no Grupo II são apontados na Figura 3, sendo a síndrome convulsiva, a encefalopatia hipóxico-isquêmica e o retardo do desenvolvimento neuropsicomotor os diagnósticos mais freqüentes.

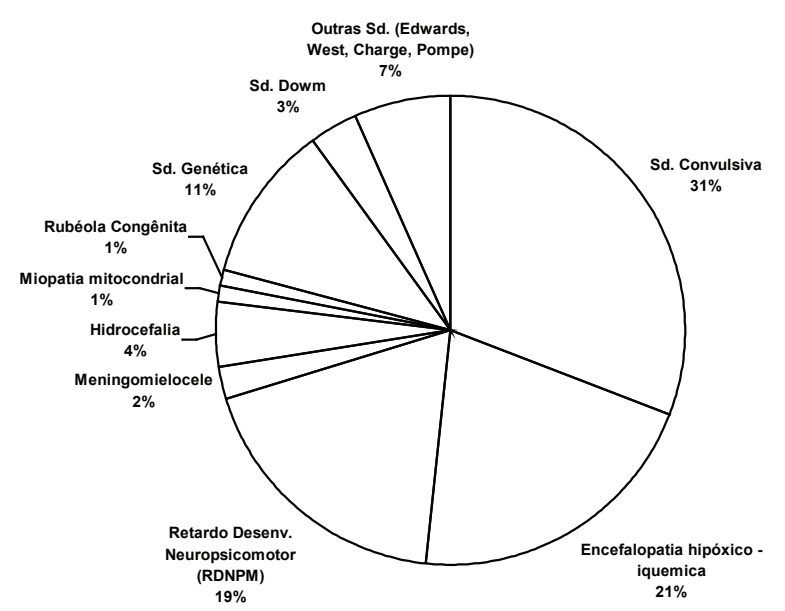

FIGURA 3 - Distribuição dos pacientes do Grupo II, segundo o diagnóstico das alterações neurológicas.

\section{Evolução ponderal}

A proporção de pacientes na curva de evolução pôndero-estatural, no percentil menor do que 2,5, foi maior no Grupo II $(P=0.01)$. A distribuição dos pacientes com DRGE, segundo a curva de evolução ponderal está demonstrada na Figura 4. 


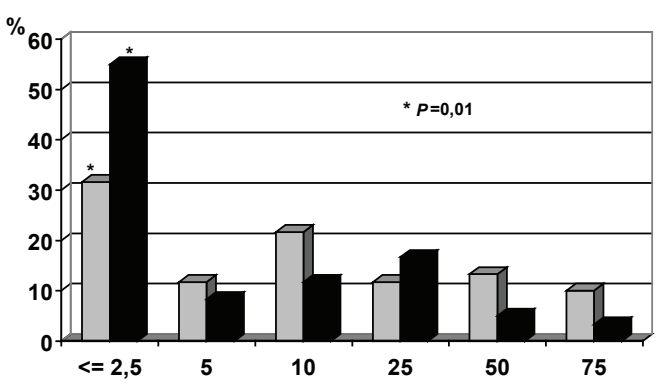

$\square \mathrm{GI}$

GIII

FIGURA 4 - Distribuição dos pacientes com refluxo, segundo a curva de evolução ponderal

Exames diagnósticos e tempo de tratamento clínico prévio à operação

Os exames complementares que mais foram utilizados no auxílio diagnóstico e na indicação cirúrgica, além da clínica apresentada pelos pacientes, foram: estudo contrastado do esôfago, estômago e duodeno (EED- 96,7\% Grupo I e 85\% Grupo II), endoscopia digestiva alta com biópsia ( $85 \%$ Grupo I e 70\% Grupo II), pHmetria esofagiana de 24 horas (23,3\% Grupo I e 33,9\% Grupo II) e cintilografia (21,7\% Grupo I e $25 \%$ Grupo II).

Todos os pacientes foram submetidos a tratamento clínico prévio. No Grupo I, na maioria dos pacientes, a duração do tratamento foi 6 meses a 1 ano $(42,3 \%)$, já no Grupo II, a duração foi menor do que 6 meses $(61,7 \%)$. A proporção de pacientes que teve tratamento clínico menor do que 6 meses foi maior no Grupo II $(P<0.01)$. Esses resultados estão representados na Figura 5.

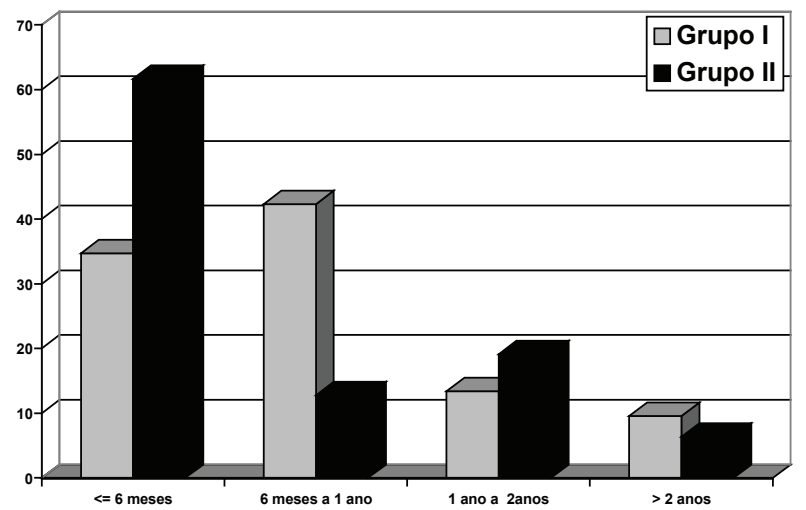

FIGURA 5 - Distribuição dos pacientes com DRGE, segundo o tempo de tratamento clínico prévio à operação

\section{Drogas utilizadas no tratamento clínico e técnicas cirúrgicas realizadas}

Além das medidas posturais e da orientação dietética, as drogas utilizadas nos dois grupos, indistintamente, foram: bloqueador H2 (cimetidina), anti-ácidos diversos, cisaprida ou metoclopramida. As operações realizadas nos dois grupos estão apresentadas na Tabela 1 .
TABELA 1 - Operações realizadas nos dois grupos de pacientes com DRGE

\begin{tabular}{lcccc}
\hline \multirow{2}{*}{\multicolumn{1}{c}{ Operações }} & \multicolumn{2}{c}{ Grupo I } & \multicolumn{2}{c}{ Grupo II } \\
\cline { 2 - 5 } & $\mathbf{n}^{\mathbf{0}}$ & $\mathbf{\%}$ & $\mathbf{n}^{\mathbf{0}}$ & $\mathbf{\%}$ \\
\hline Lind (aberta) & 37 & 61,7 & 25 & 41,7 \\
Lind + piloroplastia (aberta) & 0 & - & -2 & 3,3 \\
Lind + gastrostomia (aberta) & 3 & 5 & 33 & 55 \\
Short - Nissen (vídeo) & 20 & 33,3 & 0 & - \\
\hline
\end{tabular}

Tempo de internação e complicações no pós-operatório imediato

O tempo de internação de pacientes acometidos de DRGE foi menor no Grupo I $(P<0.001)$. A média no Grupo I foi de 5,77dias \pm 7,38 e no Grupo II foi 12,37 dias $\pm 13,60$. Os resultados estão apresentados na Figura 6.

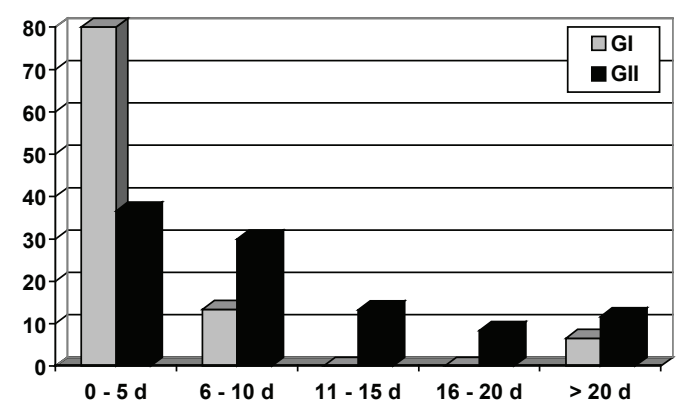

FIGURA 6 - Distribuição dos pacientes acometidos de DRGE, segundo o tempo de internação

Um total de $41,6 \%$ dos pacientes do Grupo Ie 38,3\% do Grupo II apresentaram algum tipo de complicação no pós-operatório imediato. Os resultados estão apresentados na Figura 7.

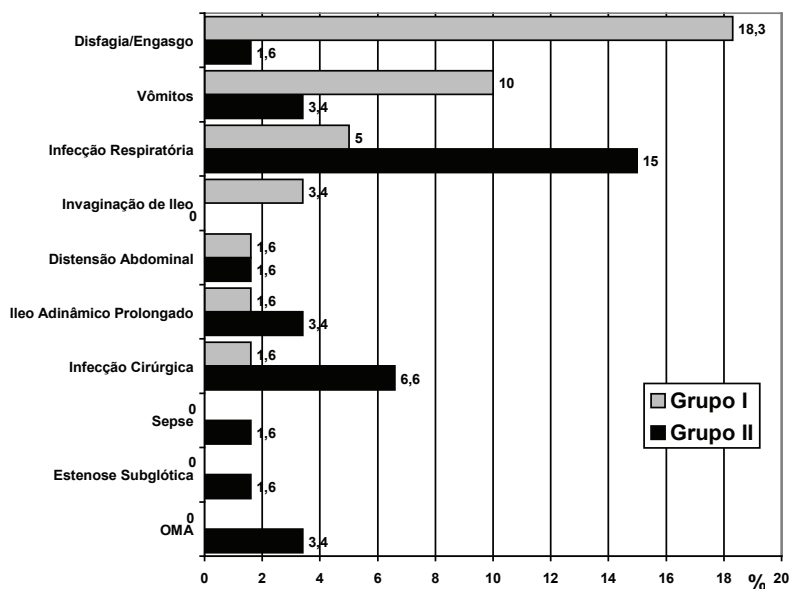

FIGURA 7 - Complicações no pós-operatório imediato nos dois grupos de pacientes com DRGE

\section{Complicações no pós-operatório tardio e reoperações}

Com relação ao pós-operatório tardio, $63,3 \%$ dos pacientes do Grupo I e 50\% do Grupo II apresentaram pelo menos algum tipo de sintomatologia, sendo que a principal 
foi a persistência dos sintomas respiratórios, em variados níveis de gravidade (38,3\% Grupo I e 47,5\% Grupo II). O resultado geral está demonstrado na Figura 8.

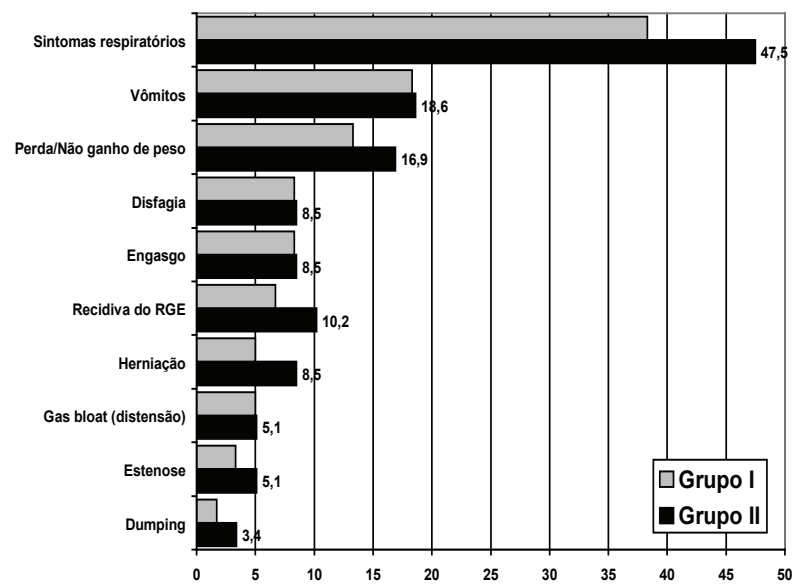

FIGURA 8 - Complicações apresentados no pós-operatório tardio nos dois grupos de pacientes com DRGE

Reoperação foi necessária em três pacientes do Grupo I $(5 \%)$ e em 11 pacientes Grupo II (18,3\%), devido à persistência da sintomatologia e/ou herniação da válvula.

A dilatação esofágica foi indicada em pacientes que apresentaram sintomas disfágicos no pós-operatório tardio, sendo 5 (8,3\%) do Grupo I e $7(11,6 \%)$ do Grupo II.

Ocorreram três óbitos no Grupo II, sendo que um foi no pós-operatório imediato devido à septicemia e dois no pós-operatório tardio devido à insuficiência respiratória e septicemia, respectivamente. Não se registraram óbitos no Grupo I. Os dados estão listados na Tabela 2.

TABELA 2 - Reoperações, dilatações esofágicas e óbitos nos dois grupos de pacientes com DRGE

\begin{tabular}{lcccc}
\hline \multirow{2}{*}{ Reoperações } & \multicolumn{2}{c}{ Grupo I } & \multicolumn{2}{c}{ Grupo II } \\
\cline { 2 - 5 } & $\mathbf{n}$ & $\mathbf{\%}$ & $\mathbf{n}$ & $\mathbf{\%}$ \\
\hline Reoperação & 3 & 5 & 11 & 18,3 \\
Reconstrução de válvula & 2 & 3,3 & 7 & 11,6 \\
Hiatoplastia & 1 & 1,6 & 3 & 5,0 \\
Rafia de esôfago abdominal & 1 & 1,6 & 0 & - \\
Gastrostomia & 0 & - & 4 & 6,6 \\
Piloroplastia & 0 & - & 1 & 1,6 \\
Dilatação esofágica & 5 & 8,3 & 7 & 11,6 \\
Óbitos & 0 & - & 3 & 5,0 \\
\hline
\end{tabular}

\section{DISCUSSÃO}

Diversos autores apontam que a causa da DRGE é de origem multifatorial. Teorias para tentar explicar a alta incidência de DRGE em neuropatas inclui a redução da pressão do esfíncter esofágico inferior, a dismotilidade esofágica e a compressão gástrica por escoliose ou espasticidade da musculatura abdominal, além da influência de efeitos posturais (posição supina predominante) e o atraso no esvaziamento gástrico $0^{9,14}$.

Há evidências significativas em estudos experimentais de que o sistema nervoso central (SNC) pode estar relacionado com a função e a motilidade gastroesofágica. $\mathrm{O}$ SNC controla a motilidade do esôfago distal e também uma parte do mecanismo de deglutição. Estudos experimentais com gatos têm mostrado que a elevação da pressão média intracraniana causa redução na pressão do esfíncter esofágico inferior. Uma vez disfuncionalizado, ele não recupera sua função normal. O envolvimento difuso do SNC na peristalse esofágica e a função do esfíncter inferior em animais sugerem que até mesmo leves disfunções do SNC podem se manifestar em alterações da motilidade esofágica e na piora do refluxo gastroesofágico ${ }^{8,17}$.

Os neuropatas são freqüentemente acometidos por doenças que ocorrem de forma concomitante, como as anomalias traqueoesofageanas, a asma e diversas malformações congênitas.

O refluxo gastroesofágico pode ser um sinal precoce de doença do SNC. Desta forma, as crianças que não respondem à terapia convencional anti-refluxo ou que manifestam complicações relacionadas à doença devem ser observadas com cuidado, suspeitando-se de provável doença do SNC, como base para as manifestações.

O "status" neurológico é o maior fator preditivo de sucesso ou de falha de operações anti-refluxo nas crianças $^{13}$.

Segundo Dedinsky et al. ${ }^{4}$ os pacientes neuropatas possuem alta incidência de herniação de válvula, reoperações e mortalidade.

Nos dois grupos estudados houve predominância da DRGE em lactentes. Porém, no Grupo I houve maior número de lactentes mais velhos (entre 1 e 2 anos) do que no Grupo II (entre 6 meses e 1 ano). Esta diferença se dá, provavelmente, por determinação do momento da indicação cirúrgica, que é mais precoce em pacientes neuropatas, em função da gravidade e da exacerbação dos sintomas apresentados.

Dentre os sintomas predominantes nos dois grupos chamou a atenção a presença de pneumonias de repetição em $90 \%$ dos pacientes do Grupo II (no Grupo I elas ocorreram em 56,7\%). Outros sintomas, como: vômito, regurgitação, engasgo, tosse noturna, irritabilidade e cianose, apesar de bastante freqüentes, apareceram de forma indistinta nos dois grupos estudados.

Com relação ao estado nutricional dos pacientes, houve maior predominância daqueles com percentil menor que 2,5 no Grupo II. Isto é em função desse grupo de pacientes sofrerem de forma mais contundente quadros repetitivos de infecções respiratórias, além de apresentarem graus variados de disfunção cricofaringeana e dismotilidade esofágica, que pioram e dificultam a ingestão regular de alimentos. O tempo de tratamento clínico prévio à operação também foi menor no Grupo II, provavelmente determinado pela gravidade dos pacientes, bem como pela ineficácia da terapêutica utilizada no alívio dos sintomas nesse grupo de doentes.

São diversas as controvérsias acerca da melhor técnica de fundoplicatura no tratamento da DRGE em crianças neuropatas. Para alguns autores, a realização da de Nissen não é a melhor escolha, uma vez que é alta a incidência 
de obstrução do intestino delgado, hérnias paraesofágicas e ruptura da válvula ${ }^{11,13,16}$. Reforçam a idéia de que a gastrofundoplicatura à Lind oferece vantagens substanciais sobre outras técnicas por permitir a liberação do excesso de pressão intra-gástrica retrogradamente. Além disso, é menos propensa à ruptura da válvula, tem menor risco de causar hérnia hiatal pós-operatória e de obstrução do intestino delgado ${ }^{15}$. Na experiência dos autores deste artigo, todos os casos de crianças neuropatas foram submetidos a procedimento de Lind (com ou sem outros procedimentos concomitantes).

Diversos autores referem a realização freqüente de gastrostomias associadas às fundoplicaturas, em pacientes neuropatas. No presente estudo, a gastrostomia foi realizada em $33(55 \%)$ dos pacientes neuropatas e em apenas três $(5 \%)$ dos pacientes não neuropatas.

Segundo Johnson ${ }^{9,10}$ a gastrostomia usualmente realizada não faz parte da operação anti-refluxo, devendo ser indicada em pacientes com lesões neurológicas graves que tenham distúrbios de desnutrição e em pacientes com desenvolvimento pôndero-estatural comprometido. Este procedimento visa a permitir a introdução de dieta adequada e, posteriormente, a dilatação do esôfago por meio da introdução de um fio guia, nos pacientes com estenose do esôfago por comprovação endoscópica² .

A piloroplastia é indicada quando há retardo no esvaziamento gástrico ${ }^{5}$; nesta casuística foi realizada em 3,3\% dos pacientes neuropatas (dois casos).

O tempo de internação dos pacientes do Grupo II foi bem maior que o do Grupo I, principalmente pela gravidade das complicações pós-operatórias, onde a incidência de afecções respiratórias e infecções cirúrgicas apareceram de forma destacada ( $15 \%$ e $6,6 \%$ respectivamente).

A persistência dos sintomas respiratórios, de forma exacerbada, foi um dos resultados que mais desapontaram a expectativa de diversos autores, acerca da eficácia do tratamento cirúrgico da DRGE, em crianças neuropatas. Robie et al. ${ }^{15}$ afirmaram que apenas $31 \%$ dos pacientes que apresentam sintomas respiratórios no período préoperatório, tornam-se assintomáticos após a operação. Neste estudo, os sintomas respiratórios foram as principais causas de complicações tardias, em ambos os grupos estudados, com predominância no Grupo II $(47,5 \%)$.

Os relatos na literatura denotam que as reoperações por recidiva do refluxo gastroesofágico ocorrem entre $8 \%$ e $12 \% \%^{3,12,19}$.

Nesta casuística, o índice de reoperações foi de 11,6\%, sendo 5\% no Grupo I e 18,3\% no Grupo II. A reconstrução da válvula foi necessária em sete $(11,6 \%)$ pacientes neuropatas e em apenas dois $(3,3 \%)$ sem neuropatia concomitante.

$\mathrm{O}$ índice de complicações ligadas à fundoplicatura foi semelhante nos dois grupos estudados. A mortalidade pósoperatória foi baixa, ocorrendo apenas três óbitos, todos no Grupo II (5\%), e devida a causas de ordem clínica.

O aumento da mortalidade no Grupo II pode refletir a alta incidência de quadros debilitantes concomitantes nesses pacientes.

\section{CONCLUSÃO}

Os resultados da fundoplicatura nos dois grupos estudados demonstram que essa opção cirúrgica é satisfatória para as crianças com DRGE que não respondem ao tratamento clínico. Porém, a população de crianças neuropatas respondem de forma menos favorável à operação, no que se refere à taxa de mortalidade, recorrência de sintomas respiratórios, índice de reoperações e gravidade das complicações pós-operatórias.

\section{AGRADECIMENTOS}

Ao Prof. Adjunto César Tadeu Spadella do Departamento de Cirurgia e Ortopedia da Faculdade de Medicina de Botucatu - Unesp pela orientação e revisão do texto.

Machado JLM, Takegawa BK, Ortolan EVOP, Rodrigues AM, Fioravante AM. Results of surgical treatment of gastroesophageal reflux disease in children with and without concomitant neurological affections. ABCD Arq Bras Cir Dig 2007; 20(1):45-50.

ABSTRACT - Background - Children with neurological disorders present a higher incidence of reflux, and generally symptoms do not get better with clinical treatment, making surgical interventions a necessary action. Aim - To compare the results of the anti-reflux surgery in normal children and in those with neurological disorders, identifying the leading complications and causes of re-operation. Methods - One hundred and twenty children with gastroesophageal reflux were distributed into two groups of study: Group I - 60 normal children; Group II - 60 children with neurological disorders. Barited contrast of esophagus, stomach and duodenum, high endoscopy with biopsy, 24 hours esophageal pHmetry and cintilography were the tests utilized in the diagnosis and evaluation of the efficacy of the surgery. All operated patients were refractory to clinical treatment prior to surgery. The anti-reflux surgery performed was Lind fundoplication, being associated to gastrostomy in 55\% of the Group II patients. Results - In Group II, surgery was indicated earlier than Group I. Among neuropaths, their highly affected neural psyquic motor development and repeated pneumonia were the main causes of surgery indication. Hospitalization time, number of re-operations and the need for post-operation esophageal dilatation were greater in Group II. Three deaths occurred in Group II during late postoperative period (sepsis and severe respiratory infection). Conclusion - The procedure performed is satisfactory for surgical reflux treatment. However, further studies of neuropath children population with reflux are imperative as they respond less favorably to surgery with regard to mortality rates, recurrence of respiratory symptoms, re-operation rates and severity of postoperative complications.

HEADINGS - Gastroesophageal reflux. Esophagitis, peptic. Neurologic manifestations. 


\section{REFERÊNCIAS}

1. Byrne WJ, Euler AR, Ashcraft E, Nash DG, Seibert JJ, Golladay ES. Gastroesophageal reflux in the severely retarded children who vomit: criteria for and results of surgical intervention in twenty-two patients. Surgery 1982; 91:9598.

2. Capella MR, Goldberg P, Quaresma ER, de Araujo E, Pereima ML, de Souza JA, Scherer CA, de V Normande Filho H, Takano SK, Vieira A. Refluxo gastroesofágico na criança. Tratamento cirúrgico pela técnica de Nissen. Rev Ass Med Bras 1995; 41:337-342.

3. Dalla Vecchia LK, Grosfeld JL, West KW, Rescorla FJ, Scherer LR 3rd, Engum AS. Reoperation after Nissen fundoplication in children with gastroesophageal reflux. Ann Surg 1997; 226:315-323.

4. Dedinsky GK, Vane DW, Black T, Turner MK, West KW, Grosfeld JL. Complications and reoperation after Nissen fundoplication in childhood. Ann J Surg 1987; 153:177-183.

5. Fonkalsrud EW, Foglia RP, Ament ME, Berquist W, Vargas J. Operative treatment for the gastroesophageal reflux syndrome in children. J Pediatr Surg 1989; 24:525-529.

6. Halpern LM, Jolley SG, Johnson DG. Gastroesophageal reflux. A significant association with central nervous system disease in children. J Pediatr Surg $1991 ; 26: 171-173$.

7. Hillemeier AC, Grill BB, McCallum R, Gryboski J. Esophageal and gastric motor abnormalites in gastroesophageal reflux during infancy. Gastroenterology 1983; 84:741-746.

8. Jean A. Brainstem organization of the swallowing network. Brain Bahav Evol 1984; 25:109-116.

9. Johnson DG, Jolley SG. Gastroesophageal reflux in infants and children: Recognition and treatment. Surg Clin North Am 1981; 61:1101-1151.
10. Johnson DG. The Nissen Fundoplication. In: Ashcraftrw, Holder TM eds. Pediatric Esophageal Surgery, Orlando, Grone \& Stratiton, 1986; P. 181-191.

11. Jolley SG, Tunell WP, Hoelzer DJ, Smith EI. Postoperative small bowel obstruction in children following Nissen fundoplication. J Pediatr Surg 1986; 21:407-409.

12. Kazerooni NL, VanCamp J, Hirschl RB, Drongowski RA, Coran AG. Fundoplication in 160 children under 2 years of age. J Pediatr Surg 1994; 29:677691.

13. Pearl RH, Robie DK, Ein SH, Shandling B, Wesson DE, Superina R, Mctaggart K, Garcia VF, O'Connor JA, Filler RM. Complications of gastroesophageal antireflux surgery in neurologically impaired versus neurologically normal children. J Pediatr Surg 1990; 25:1169-1173.

14. Rice H, Seashore JH, Toulloukian RJ. Evaluation of Nissen fundoplication in neurologically impaired children, J Pediatr Surg 1991; 26:697-701.

15. Robie DR, Pearl RH. Modified Nissen fundoplication: improved results in high risk children. J Pediatr Surg 1991; 26:1268-1272.

16. Smith CD, Othersen HB Jr, Gogan NJ, Walker JD. Nissen fundoplication in children with profound neurologic disability. Ann Surg 1992; 215:654-658.

17. Vane DW, Shiffler M, Grosfeld JL, Hall P, Angelides A, Weber TR, Fitzgerald JF. Reduced lower esophageal sphincter (LES) pressure after acute and chronic brain injury. J Pediatr Surg 1982; 17:960-963.

18. Wesley JR, Coran AG, Sarahan TM, Klein MD, White SJ. The need for evaluation of gastroesophageal reflux in brain - damaged children referred for feeding gastrostomy. J Pediatr Surg 1981; 16:866-871.

19. Wheatley MJ, Coran AG, Wesley JR, Oldham KT, Turnage RH. Redo fundoplication in infants and children with recurrent gastroesophageal reflux. J Pediatr Surg 1991; 26:758-761.

Conflito de interesse: não há

Fonte financiadora: não há

Recebido para publicação em: 05/09/2006

Aceito para publicação em: 03/12/2006 